\title{
A Cell-Capture and Release Platform Based on Peptide-Aptamer-modified Nanowires
}

\author{
Jingying Li, ${ }^{[-]}$Cui Qi,${ }^{[-]}$Zheng Lian, Qiusen Han, Xinhuan Wang, Shuangfei Cai, \\ Rong Yang,* Chen Wang*
}

CAS Key Lab for Biological Effects of Nanomaterials and Nanosafety, National Center for Nanoscience and Technology, Beijing 100190, P. R. China

\footnotetext{
*Corresponding author: yangr@ nanoctr.cn, wangch@ nanoctr.cn

${ }^{[-]}$These authors are equally contributed.
}

\section{This file contains:}

Figure S1. SEM image and SAED pattern of GaN nanowires (NWs).

Figure S2. XPS spectra of three GaN NW surfaces: the one before and after peptide P1 absorption, and the one modified with P1-S2.2.

Figure S3. WCA measurements of three GaN NW surfaces: the one before and after peptide P1 absorption, and the one modified with P1-S2.2.

Figure S4. Raman spectra of three GaN NW surfaces: the one before and after peptide P1 absorption, and the one modified with P1-S2.2.

Figure S5. Confocal microscope images of MCF-7 cells captured on GaN NW surfaces.

Figure S6. MCF-7 Cells captured on P1-S.2.2-modified GaN NW surfaces with and without FBS.

Figure S7. MCF-7 cell-capture yields with different aptamer S2.2 concentrations and at different capture times.

Figure S8. Fluorescence microscopy images of A549 and Jurkat cells captured on GaN NW surfaces, and the numbers of both captured cells.

Figure S9. Controlled release of captured MCF-7 cells from the GaN NW surfaces utilized DNAase at different reaction time.

Figure S10. Fluorescence microscope images of MCF-7 cells captured on GaN NW surface, released and cultured for different generations.

Figure S11. Confocal microscopy images of MCF-7 cells cultured for third generation after release from the GaN NW surface. 


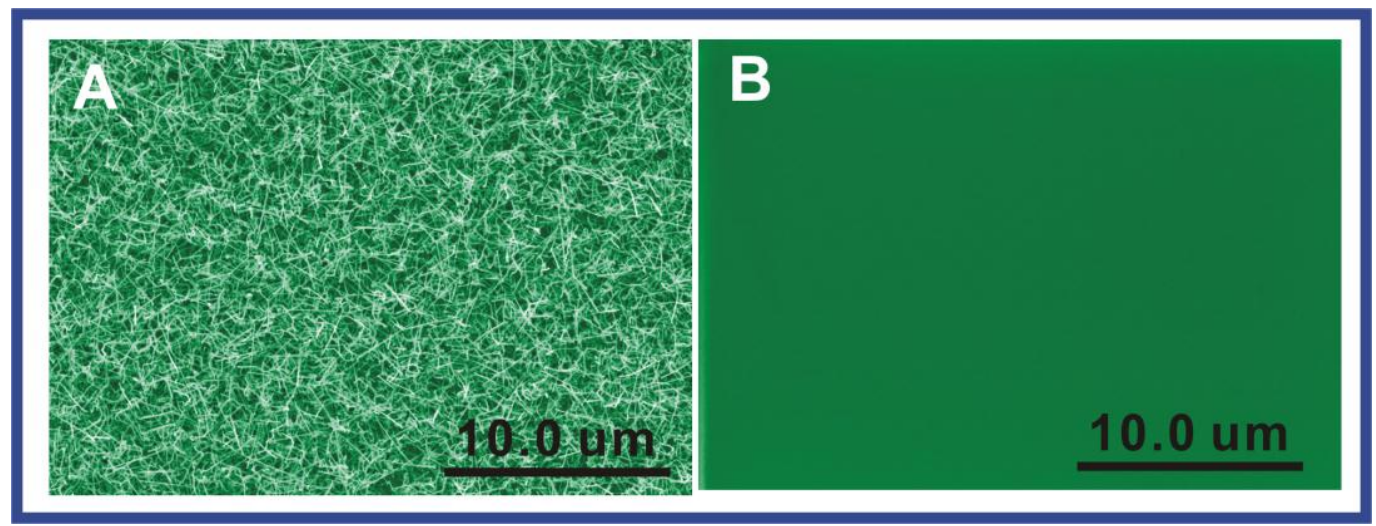

Figure S1. Large scale SEM images of GaN NW surface (A) and flat surface (B).
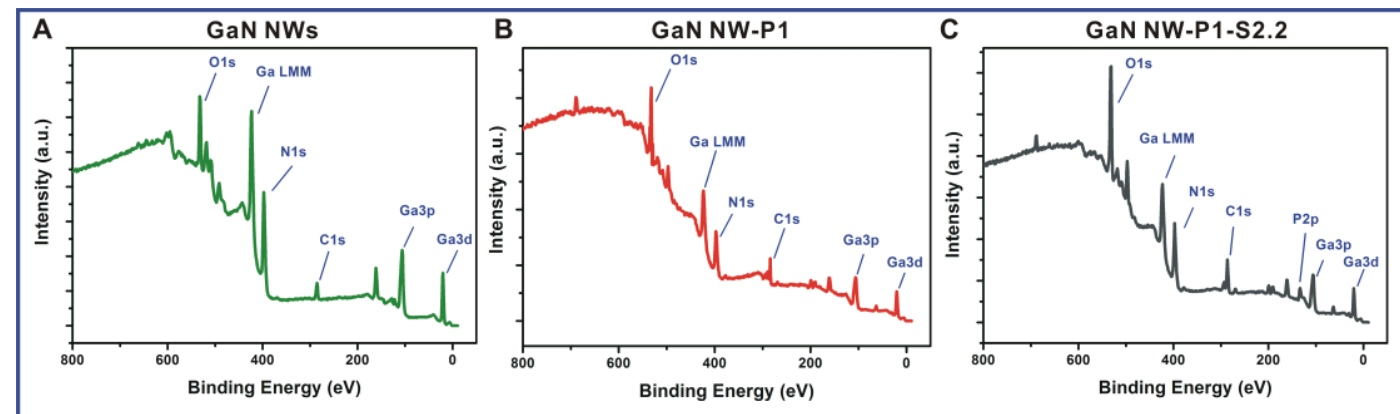

Figure S2. XPS spectra of three GaN NW surfaces: (A) and (B) are the one before and after peptide P1 absorption, respectively; (C) the one modified with P1-S2.2. The ratio of N1s /Ga3d: GaN-P1-S2.2>GaN-P1>GaN; the ratio of O1s /Ga3d: GaN-P1-S2.2>GaN-P1>GaN; the ratio of C1s /Ga3d: GaN-P1-S2.2>GaN-P1>GaN

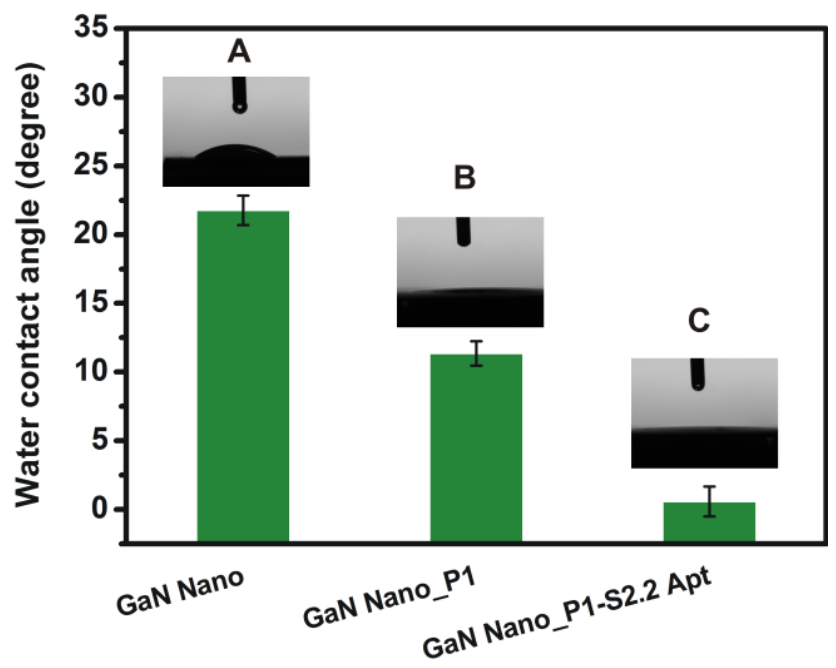

Figure S3. Water contact angle (WCA) measurements of three GaN NW surfaces: (A) the one before peptide $\mathrm{P} 1$ absorption; (B) the one after peptide $\mathrm{P} 1$ absorption; (C) the one modified with P1-S2.2. 


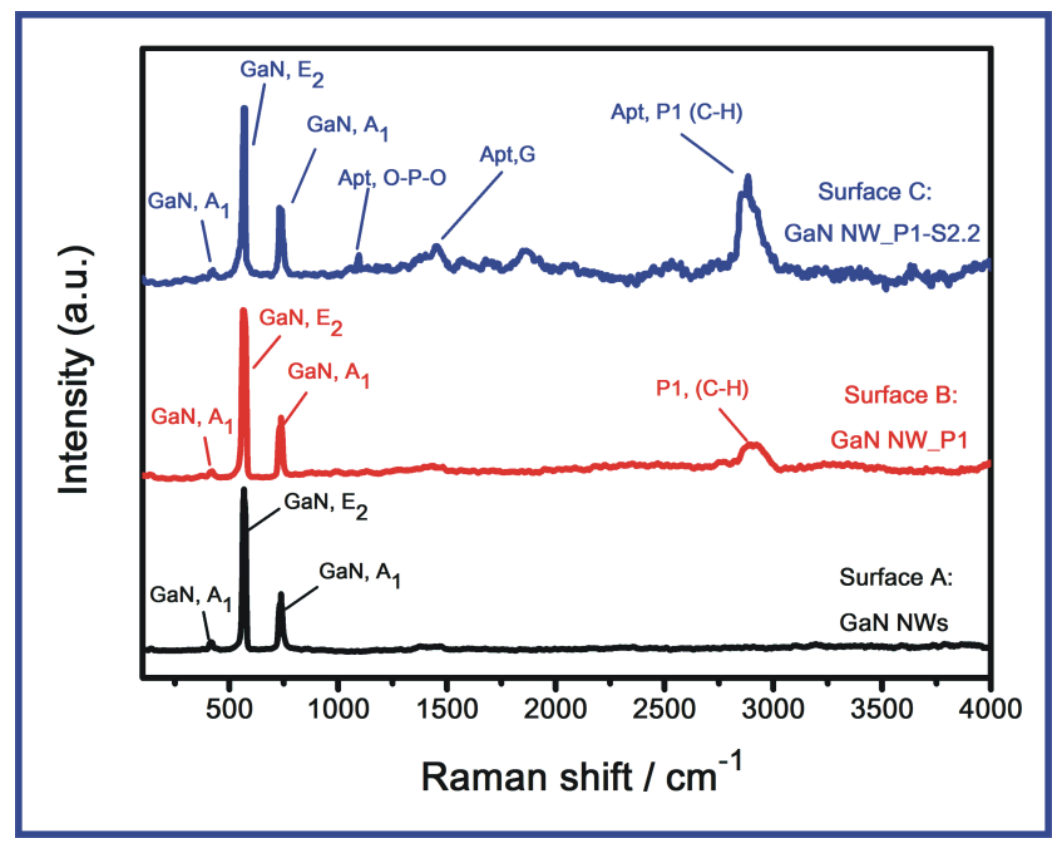

Figure S4. Raman spectra of three GaN NW surfaces: (A) the one before peptide P1 absorption; (B) the one after peptide P1 absorption; (C) the one modified with $\mathrm{P} 1-\mathrm{S} 2.2$.

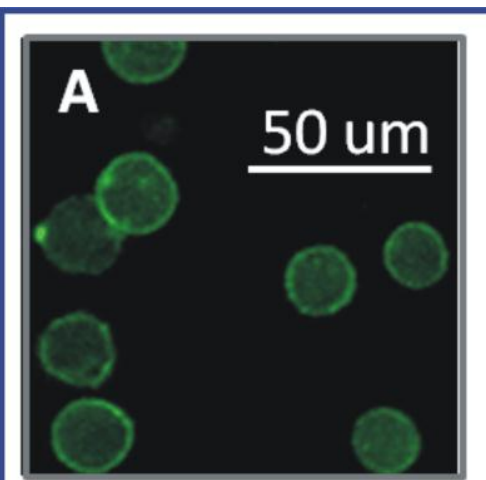

FITC-PA

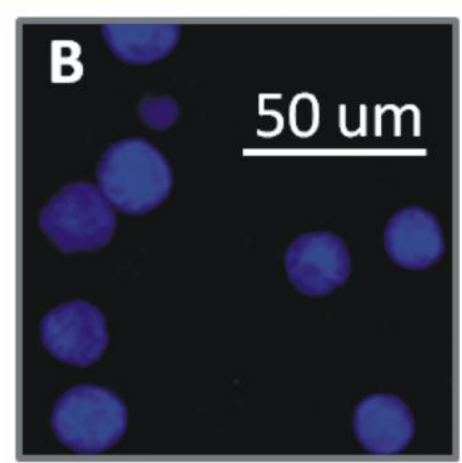

DAPI

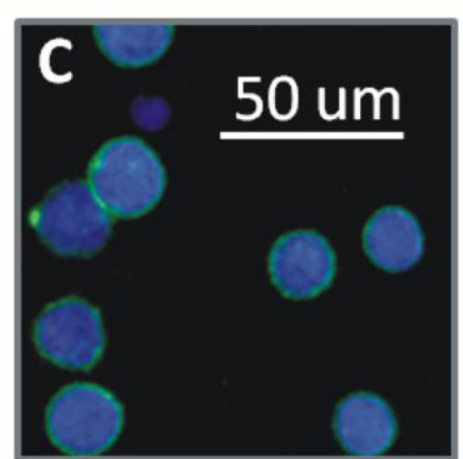

Merged

Figure S5. Confocal microscope images of MCF-7 cells captured on the GaN NW surface which was modified with P1-S2.2: (A) actin stained by FITC-phalloidin, (B) nucleus stained by DAPI, (C) Merged. 


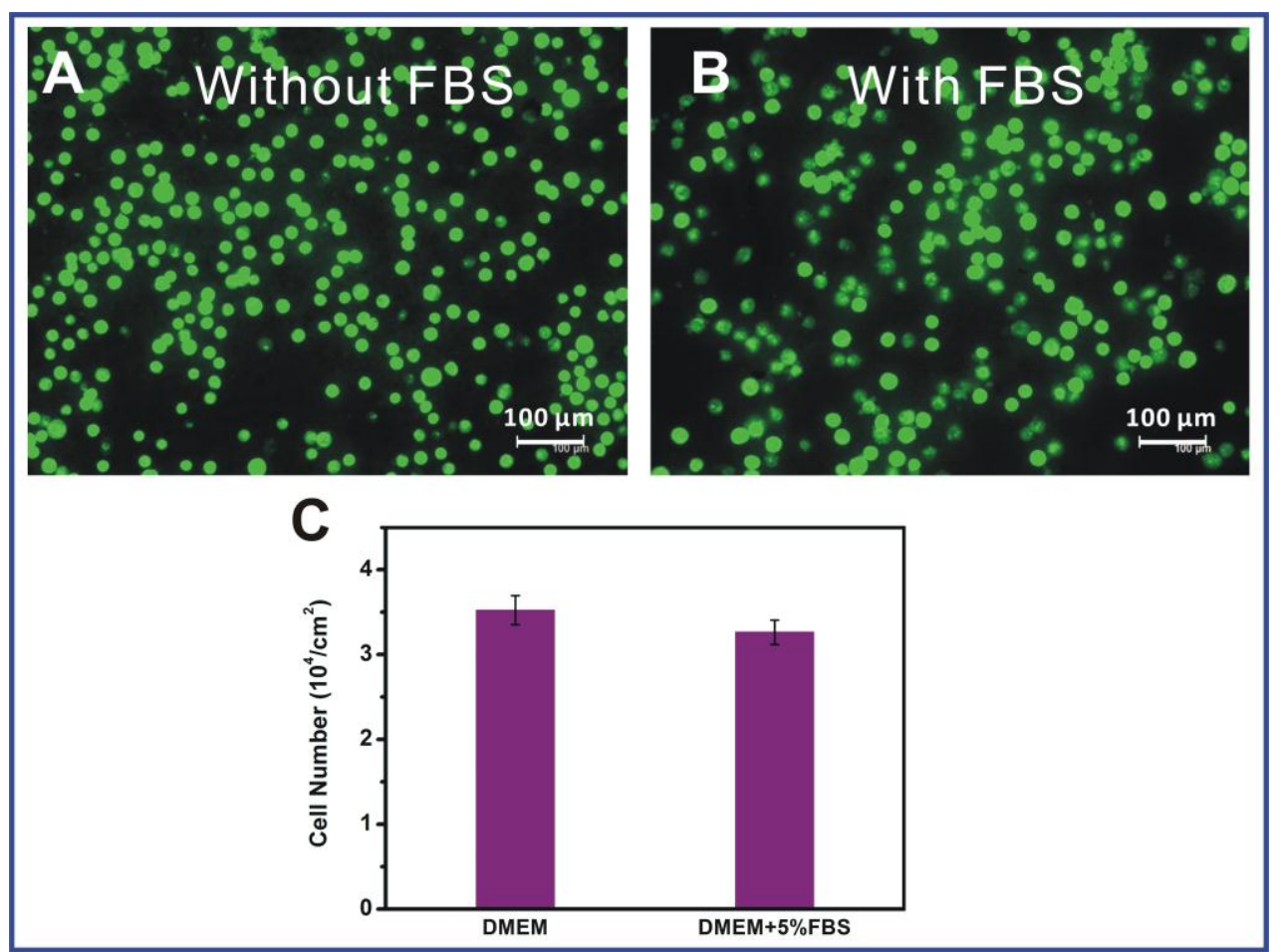

Figure S6. MCF-7 Cells captured on P1-S.2.2-modified GaN NW surfaces (A) without and (B) with fetal bovine serum (FBS), (C) The cell numbers on these two surfaces.

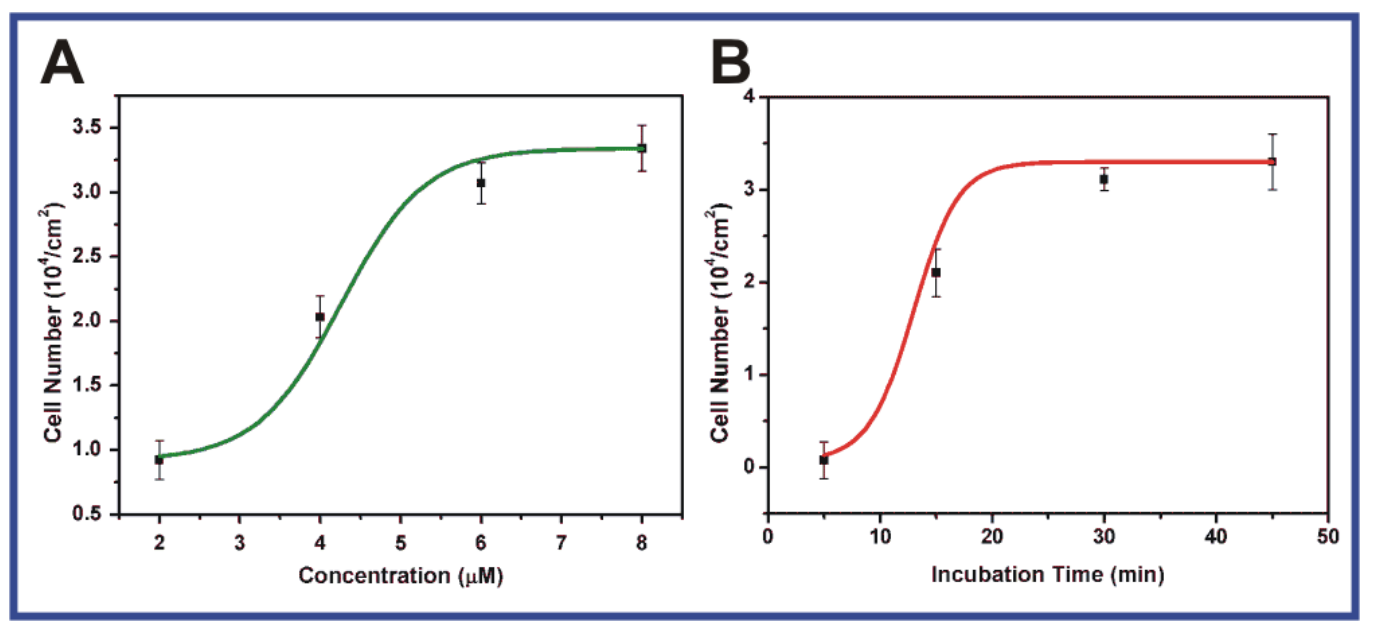

Figure S7. MCF-7 cell-capture yields with (A) different aptamer S2.2 concentrations and (B) at different capture times. 


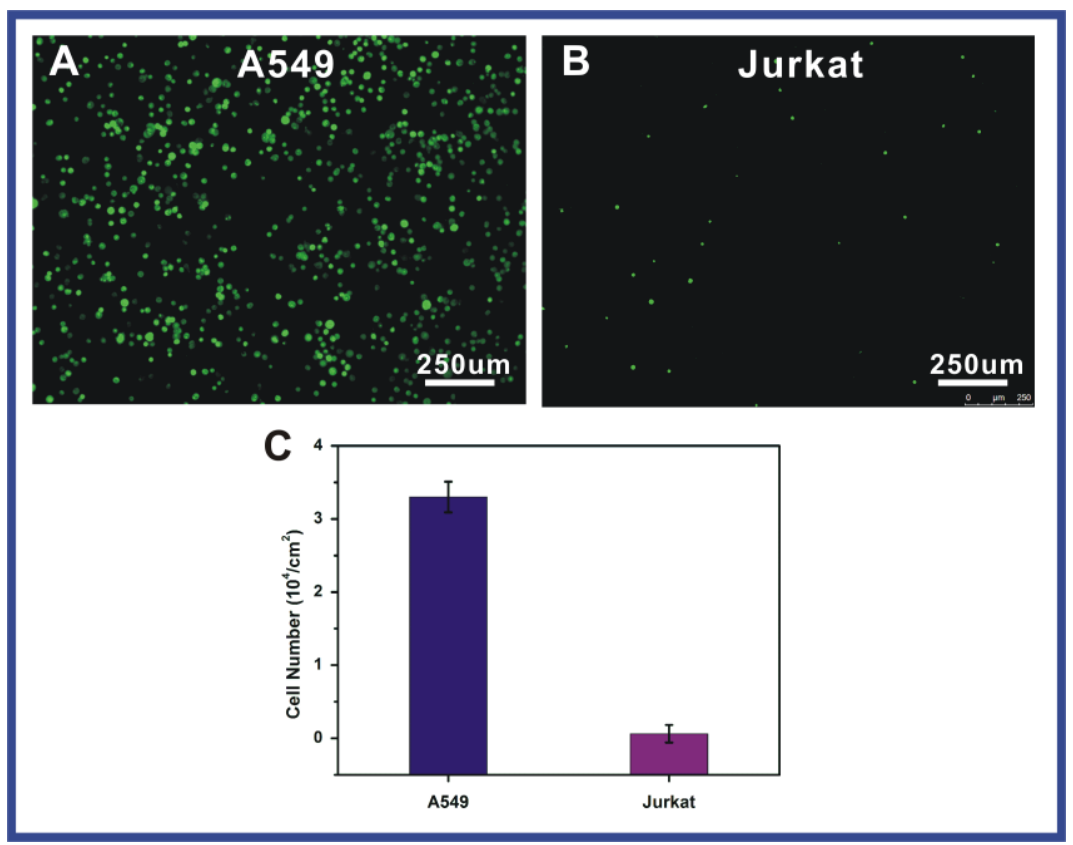

Figure S8. (A) and (B) are fluorescence microscopy images of A549 cells (MUC1-positive cell line) and Jurkat cells (MUC1-negetive cell line) captured on P1-S.2.2-modified GaN NW surfaces; (C) The numbers of both captured cells.

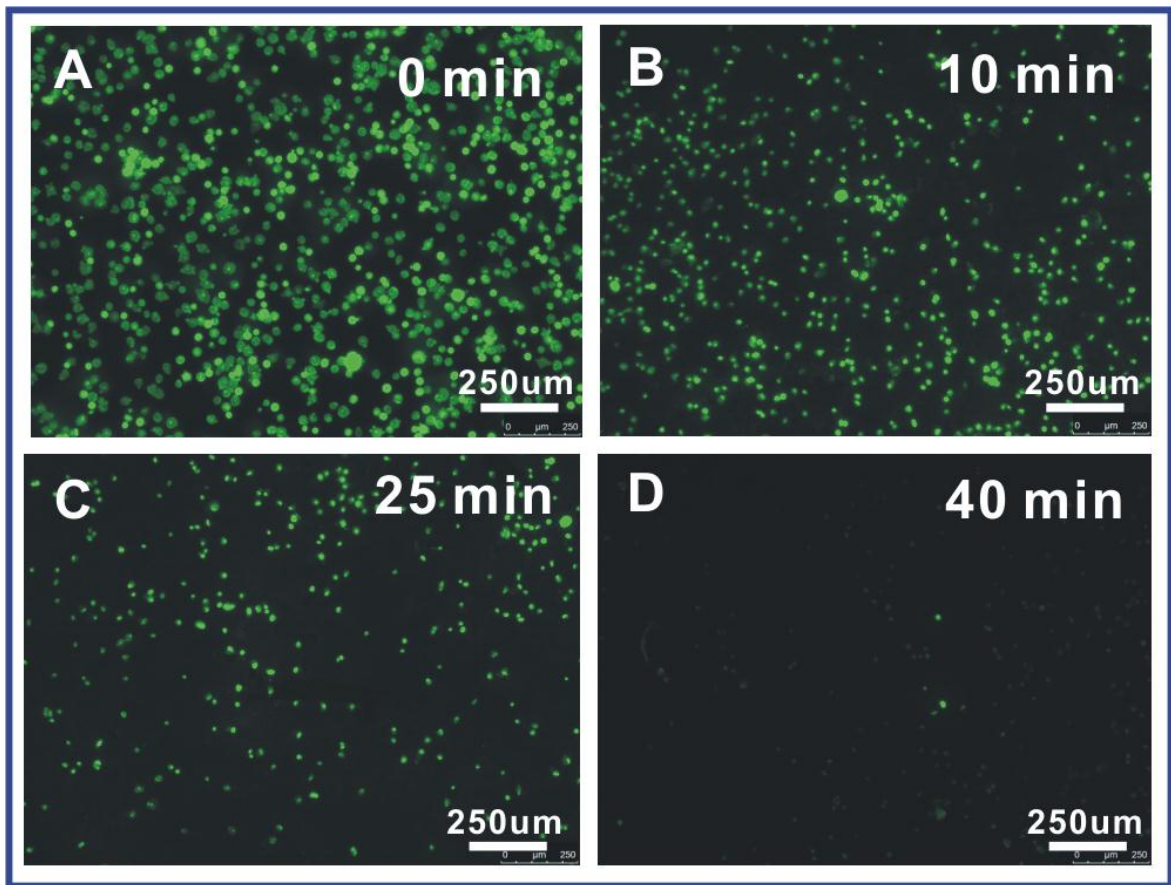

Figure S9. Controlled release of captured MCF-7 cells from the P1-S.2.2-modified GaN NW surfaces utilized DNAase at different reaction time: (A) $0 \mathrm{~min}$; (B) $10 \mathrm{~min}$; (C) $25 \mathrm{~min}$; (D) $40 \mathrm{~min}$. 


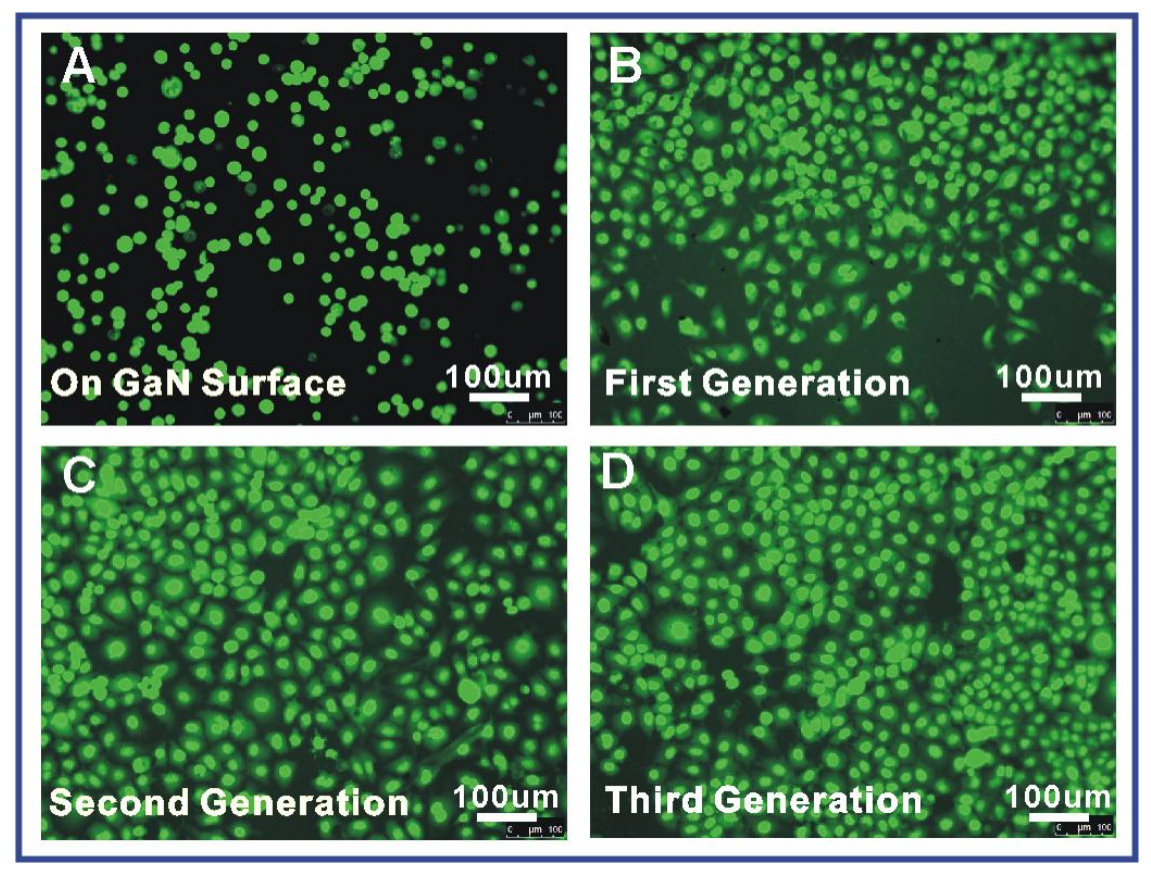

Figure S10. Fluorescence microscope images of MCF-7 cells: (A) captured on P1-S.2.2-modified GaN NW surface; (B) released and cultured for first generation (after $24 \mathrm{~h}$ ); (C) released and cultured for second generation (after $48 \mathrm{~h}$ ); (D) released and cultured for third generation (after $36 \mathrm{~h}$ ).

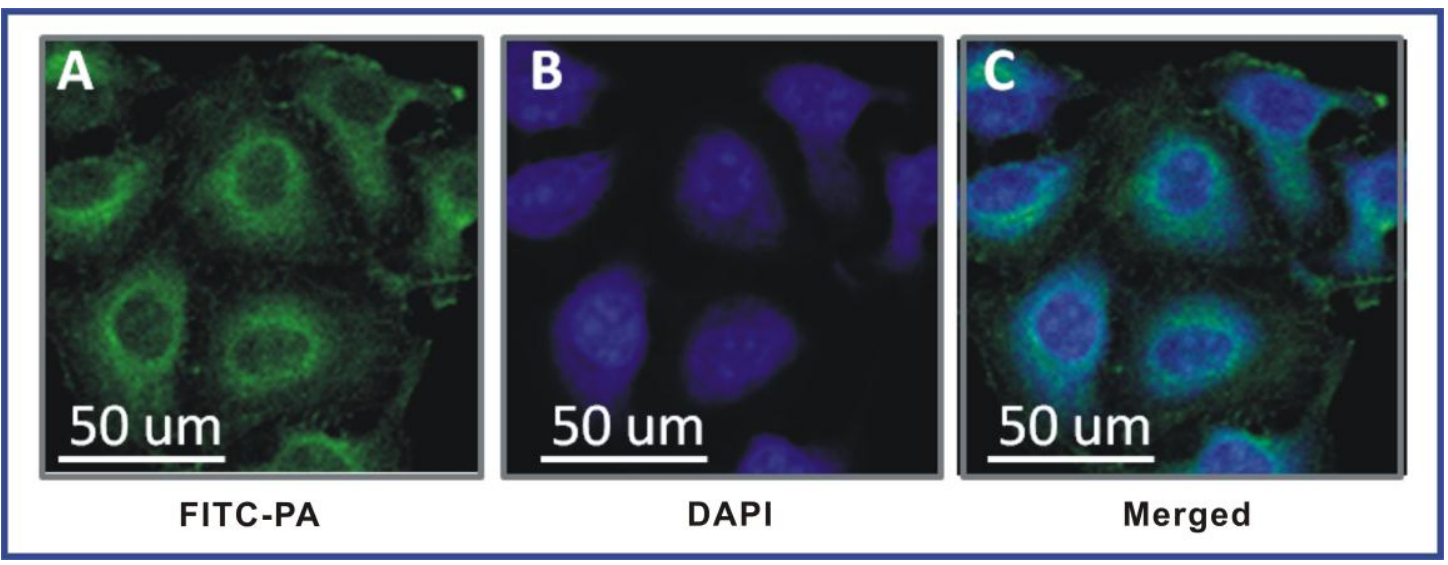

Figure S11. Confocal microscope images of MCF-7 cells cultured for third generation (after $36 \mathrm{~h}$ ) after release from the GaN NW surface. (A) actin stained by FITC phalloidin; (B) nucleus stained by DAPI; (C) Merged. 